BMJ Open

Diabetes

Research

\& Care

\section{Genistein stimulates insulin sensitivity through gut microbiota reshaping and skeletal muscle AMPK activation in obese subjects}

To cite: Guevara-Cruz M, Godinez-Salas ET, Sanchez-Tapia M, et al. Genistein stimulates insulin sensitivity through gut microbiota reshaping and skeletal muscle AMPK activation in obese subjects. BMJ Open Diab Res Care 2020;8:e000948. doi:10.1136/ bmjdrc-2019-000948

- Additional material is published online only. To view, please visit the journal online (http://dx.doi.org/10.1136/ bmjdrc-2019-000948).

Received 10 0ctober 2019 Revised 26 November 2019 Accepted 20 December 2019

Check for updates

C Author(s) (or their employer(s)) 2020. Re-use permitted under CC BY-NC. No commercial re-use. See rights and permissions. Published by BMJ.

For numbered affiliations see end of article.

Correspondence to Dr Armando R Tovar; tovar.ar@gmail.com

\section{ABSTRACT}

Objective Obesity is associated with metabolic abnormalities, including insulin resistance and dyslipidemias. Previous studies demonstrated that genistein intake modifies the gut microbiota in mice by selectively increasing Akkermansia muciniphila, leading to reduction of metabolic endotoxemia and insulin sensitivity. However, it is not known whether the consumption of genistein in humans with obesity could modify the gut microbiota reducing the metabolic endotoxemia and insulin sensitivity.

Research design and methods 45 participants with a Homeostatic Model Assessment (HOMA) index greater than 2.5 and body mass indices of $\geq 30$ and $\leq 40 \mathrm{~kg} / \mathrm{m}^{2}$ were studied. Patients were randomly distributed to consume (1) placebo treatment or (2) genistein capsules (50 mg/ day) for 2 months. Blood samples were taken to evaluate glucose concentration, lipid profile and serum insulin. Insulin resistance was determined by means of the HOMA for insulin resistance (HOMA-IR) index and by an oral glucose tolerance test. After 2 months, the same variables were assessed including a serum metabolomic analysis, gut microbiota, and a skeletal muscle biopsy was obtained to study the gene expression of fatty acid oxidation.

Results In the present study, we show that the consumption of genistein for 2 months reduced insulin resistance in subjects with obesity, accompanied by a modification of the gut microbiota taxonomy, particularly by an increase in the Verrucomicrobia phylum. In addition, subjects showed a reduction in metabolic endotoxemia and an increase in 5 -adenosine monophosphate-activated protein kinase phosphorylation and expression of genes involved in fatty acid oxidation in skeletal muscle. As a result, there was an increase in circulating metabolites of $\beta$-oxidation and $\omega$-oxidation, acyl-carnitines and ketone bodies.

Conclusions Change in the gut microbiota was accompanied by an improvement in insulin resistance and an increase in skeletal muscle fatty acid oxidation. Therefore, genistein could be used as a part of dietary strategies to control the abnormalities associated with obesity, particularly insulin resistance; however, long-term studies are needed.

\section{Significance of this study}

What is already known about this subject?

- Obesity is known to be associated with dysbiosis of the intestinal microbiota, which in turn is accompanied by impaired insulin sensitivity.

What are the new findings?

-We found that genistein improved insulin sensitivity by increasing phosphorylation of the enzyme adenosine monophosphate protein kinase in the skeletal muscle of obese subjects.

- The study also showed that treatment with genistein decreased gut microbiota dysbiosis and metabolic endotoxemia and stimulated the abundance of Akkermansia muciniphila, which may also contribute to improve insulin sensitivity

How might these results change the focus of research or clinical practice?

- Genistein can be used as an additional strategy to decrease insulin resistance in obese subjects.

\section{INTRODUCTION}

Epidemiological studies in several parts of the world have shown an alarming increase in the epidemics of obesity. ${ }^{1}$ This situation has been accompanied by an increase in metabolic abnormalities, mainly insulin resistance and dyslipidemias that have increased the rate of morbimortality worldwide. $^{2}$ In order to attend to this problem, several clinical and nutritional strategies have been developed to attenuate some of the altered metabolic conditions. ${ }^{3}$ There is evidence that strategies that increase the capacity to oxidize fatty acids in the body of 
obese animals can contribute to improve insulin sensitivity and reduce the hyperlipidemia. ${ }^{4}$

Several compounds have been used to increase fatty acid oxidation and therefore insulin sensitivity ${ }^{5}$ by the activation of the enzyme $5^{\prime}$-adenosine monophosphateactivated protein kinase (AMPK). ${ }^{7}$ The increase of fatty acid oxidation prevents the accumulation of lipids in several organs, decreasing the lipotoxicity. ${ }^{8}$ In particular, the reduction in the accumulation of lipids in the skeletal muscle is accompanied by an increase in insulin sensitivity. ${ }^{9}$ Among the compounds that have been shown to increase AMPK activity is genistein, an isoflavone present in some foods, particularly in soy. ${ }^{10}$ Studies in rat skeletal muscle and adipose tissue show that phosphorylation of AMPK by genistein is accompanied by an increase in the expression of genes involved in fatty acid oxidation and also an increase in energy expenditure. ${ }^{11} 12$ In addition, C2C12 myotubes incubated with genistein have a greater capacity to oxidize palmitate. ${ }^{12}$

Interestingly, there is also evidence that AMPK activation is modified by the gut microbiota. The first studies indicated that intestinal colonization of germ-free mice with gut microbiota can modify the phosphorylation of AMPK, and it was associated with an increase in body fat accumulation and an increase in serum glucose and insulin concentration. Nonetheless, the mechanism involved in the inactivation of AMPK was not clear. ${ }^{13}$ Further findings demonstrated that metabolic abnormalities during obesity were associated with a condition named metabolic endotoxemia, in which there is an alteration on the gut microbiota due to a change in the intestinal gut permeability allowing an increase in the influx of lipopolysaccharide (LPS) ${ }^{14-16}$ However, it is not clear whether LPS could downregulate AMPK activity. Studies on the hypothalamus revealed that intraperitoneal injection of LPS decreased the phosphorylation of AMPK, as well as its target protein acetyl coenzyme A (CoA) carboxylase. ${ }^{17}$ Thus, a reduction of circulating levels of LPS could attenuate some of the metabolic alterations in obese subjects, particularly insulin resistance.

Several studies indicate that some dietary bioactive compounds can modify the gut microbiota selectively by enhancing or decreasing specific species of the gut microbiota. ${ }^{18}$ Studies with mice have demonstrated that consumption of genistein in their diet is able to modify the gut microbiota by increasing selectively Akkermansia muciniphila, and the modification of the gut microbiota was accompanied with a reduction in the circulating concentration of LPS, leading to an improvement in insulin sensitivity. ${ }^{19}$ However, it is not known whether consumption of genistein in humans could modify the gut microbiota and improve insulin sensitivity. Therefore, the aim of the present work was to assess whether supplementation with genistein modifies the gut microbiota in obese subjects with metabolic syndrome in order to decrease the serum concentration of LPS and thereby to improve the insulin sensitivity by activation of AMPK in skeletal muscle.

\section{RESEARCH DESIGN AND METHODS}

\section{Subjects and study design}

We included 45 participants who met the following inclusion criteria: adults between 20 and 60 years of age with Homeostatic Model Assessment (HOMA) index greater than 2.5, body mass indices (BMIs) of $\geq 30$ and $\leq 40 \mathrm{~kg}$ / $\mathrm{m}^{2}$ and who signed the consent letter. Patients who had any added pathology, pregnancy, smoking or consumed medications were excluded. Once the letter of informed consent was obtained, the patients were assigned to the respective treatment group. These individuals were advised to consume the recommended diet according to the guidelines of the Adult Treatment Panel III. To assess the effect of genistein on obese subjects with obesity, we designed a parallel, double-blind study (online supplementary figure $1)$. The subjects were randomly selected to form part of the placebo group (PG) or the genistein-treated group (GTG) with genistein capsules $(50 \mathrm{mg} /$ day). The participants were followed up for 2 months. In the prerandomization visit, informed consent letters were given, and blood samples were taken to evaluate glucose concentration, lipid profile and serum insulin, blood pressure, body weight and height and body composition. The presence of insulin resistance was determined by means of the HOMA for insulin resistance (HOMA-IR) index, the Matsuda index and an oral glucose tolerance test. After 2 months, the same variables were assessed, and an expert surgeon in the operating room performed a vastus lateralis muscle biopsy.

\section{Oral glucose tolerance test}

Patients underwent two glucose tolerance curves: (1) one before the consumption of placebo or genistein (baseline) and (2) the second curve, 2 months after starting treatment. Blood samples were taken and the area under the curve (AUC) of the glucose and insulin concentrations was calculated. Additionally, surrogate analyses derived from the oral glucose tolerance test were calculated. ${ }^{20}$

\section{Serum biochemical variables}

Total cholesterol, triglycerides, high-density lipoprotein (HDL) cholesterol, low-density lipoprotein cholesterol and glucose were measured in serum using COBAS c111 photometric equipment from the patients after $8-12$ hours of fasting. Leptin, adiponectin and insulin were determined by the immunoenzymatic assay (ELISA) method (ALPCO). Serum LPS was determined by ELISA kit (Cloud-Clone Corp., USA).

\section{Skeletal muscle biopsy}

A sample from the vastus lateralis muscle in the area located $25 \mathrm{~cm}$ from the tibial tuberosity and $5 \mathrm{~cm}$ from the femoral midline was obtained. A $1 \mathrm{~cm}^{3}$ portion of muscle was taken by means of a ring clamp. The biopsies were immediately placed in liquid nitrogen and stored in an ultrafreezer at $-80^{\circ} \mathrm{C}$ for further analysis.

\section{Protein extraction and western blotting}

Twenty micrograms of protein extracted from muscle was loaded onto $12 \%$ polyacrylamide gels, transferred to 
polyvinylidene difluoride (PVDF) transfer membranes (IPVH00010, Immobilon-P, Millipore) and blotted overnight at $4^{\circ} \mathrm{C}$ with the respective primary antibodies against acetyl-CoA carboxylase (ACC) (MA5-15025, Pierce Thermo Scientific), phospho-acetyl CoA carboxylase (07-303, Millipore), AMPK (NB100-239 Novus bio), pAMPK (07-681 Millipore) and glyceraldehyde 3-phosphate dehydrogenase (GAPDH) (sc-365062 Santa Cruz) and revealed with a secondary antibody conjugated to horseradish peroxidase. Bands were quantitated relative to an in-blot reference band (ThermoFisher Scientific), followed by normalization with their respective GAPDH.

\section{Mitochondrial activity and lipid content in skeletal muscle}

For succinate dehydrogenase (SDH) staining, slides of muscle biopsies were incubated in SDH staining solution according to a previous report. ${ }^{21}$ Assessment of lipid content in muscle fibers was done using the lipophilic dye boron-dipyrromethene (BODIPY) 493/503 (790389, Sigma-Aldrich). Slides were mounted with ProLong Gold Antifade Mountant with 4',6-diamidino-2-phenylindole (DAPI) (P36931, Invitrogen). Histochemical staining was quantified by ImageJ (National Institutes of Health) software.

\section{Gut microbiota}

A fecal sample from all subjects was collected before and after 2 months of treatment with placebo or genistein. Fecal samples were frozen at $-80^{\circ} \mathrm{C}$. DNA extraction was carried out using a QIAamp DNA Stool Mini Kit (Qiagen, USA) according to the manufacturer's instructions. Variable regions 3-4 of the $16 \mathrm{~S}$ rRNA gene were amplified using specific primers containing the Illumina adapter overhang nucleotide sequences. An index PCR was then carried out to attach dual indices using a Nextera XT V.2 Kit. Sequencing was performed on an Illumina MiSeq platform (MiSeq Reagent Kit V.3, 600 cycles) according to the manufacturer's instructions to generate paired-end reads of 300 bases in length in each direction.

Microbial sequence data were pooled for operational taxonomic unit (OTU) comparison and taxonomic abundance analysis but separated by batch in the principal coordinates analysis (PCoA) using Emperor. The community diversity was determined by the number of OTUs and beta diversity, measured by UniFrac unweighted and weighted distance matrices in Quantitative Insights Into Microbial Ecology (QIIME). Analysis of similarities (ANOSIM), a permutational multivariate analysis of variance, was used to determine statistically significant clustering of groups based on microbiota structure distances.

\section{Metabolite analysis}

A serum metabolite analysis was performed by Metabolon (Durham, North Carolina, USA) based on ultrahighperformance liquid chromatography-tandem mass spectrometry.

\section{RNA extraction from skeletal muscle biopsies}

Skeletal muscle was homogenized in guanidinium buffer with a polytron (PT2000; Kinematica, Lucerne, Switzerland) at the lowest setting. The homogenate was centrifuged and the resulting supernatant was layered onto a $\mathrm{CsCl}$ solution. The $\mathrm{CsCl}$ gradient was formed by centrifugation at $113000 \times \mathrm{g}$ for 18 hours at $18^{\circ} \mathrm{C}$ to yield total RNA. The RNA was precipitated and resuspended in water. The RNA was quantified by optical density at $260 \mathrm{~nm}$ and stored at $-80^{\circ} \mathrm{C}$ until use.

\section{Gene expression microarray assay}

RNA quality was assessed using the RNA 6000 Nano Kit (Agilent Technologies). RNA quantitation was measured with a Nanodrop spectrophotometer (Nanodrop Technologies) using samples with RNA integrity number (RIN) of $>6.0$. Target cDNA was prepared according to the Whole-Transcript Sense Target Labeling Protocol (P/N 703174, Thermo Fisher Scientific). The labeled cDNA product was hybridized to the GeneChip HumanGene V.2.0 ST microarray (Affymetrix). The samples were washed with low-stringency and high-stringency buffers and were stained with streptavidin-phycoerythrin using the GeneChip Fluidics Station 450 with the FS450_0002 protocol. The GeneChip Scanner 3000 7G (Affymetrix) was used to collect fluorescent signals, and Expression Console software (Affymetrix) was used to obtain intensity signal and quality data of the scanned arrays. The results were analyzed with the Partek Genomics Suite for hierarchical clustering to generate the heat map.

\section{Statistical analysis}

For the statistical analysis, the mean and the SE for the quantitative variables were obtained first, and for the qualitative variables, the mean and SE were determined as a proportion of the total population using the statistical software database (Statistical Package for the Social Sciences (SPSS)). The Shapiro-Wilk test was used to determine the normality of the data; otherwise, the results were log transformed for analysis. Paired t-test for related samples was used to compare the changes in the variables before and after each of the treatments, and an unpaired t-test was used when the treatments were compared. Statistical analysis was carried out using GraphPad Prism V.7.0.

\section{RESULTS}

\section{Study subjects met obesity criteria and insulin resistance}

As observed in online supplementary table 1 , there was no significant difference in all phenotypical and metabolic variables assessed between groups. All subjects were obese as observed by BMI, waist circumference and the elevated percentage of body fat (online supplementary table 1). In addition, all subjects showed insulin resistance according to the HOMA-IR index, hyperinsulinemia, hyperleptinemia, hypertriglyceridemia and low HDL cholesterol levels. Furthermore, the subjects had elevated concentrations of 
C reactive protein, indicative of an inflammatory condition that has been associated with obesity. ${ }^{22}$

\section{Biochemical changes after 2 months of intervention with genistein}

After 2 months of treatment with genistein (GTG) or placebo $(\mathrm{PG})$, we did not observe significant differences in some anthropometric and biochemical variables (online supplementary table 1 ). In addition, there was no significant difference in blood pressure. Interestingly, the GTG showed significantly reduced basal insulin levels by approximately $24 \%$ ( $\mathrm{p}$ value $=0.05$ ) and a reduced HOMA-IR index by approximately $28 \%$ compared with the PG ( $p$ value $=0.05$ ). Additionally, the GTG presented lower $\mathrm{C}$ reactive protein levels than the $\mathrm{PG}$ ( $\mathrm{p}$ value $=0.06$ ), indicative of a reduction in the proinflammatory state.

\section{Serum metabolite signature of genistein consumption}

In the GTG, the circulating metabolite profile showed that the levels of 30 circulating metabolites differed from those in the PG according to the random forest analysis $(80 \%$ accuracy) (online supplementary figure 2). The metabolites included several amino acids or molecules associated with amino acid metabolism, lipids, such as phospholipids and dicarboxylates, and some unknown metabolites.

\section{Genistein modifies the gut microbiota and reduces metabolic endotoxemia}

We assessed whether genistein could modify the gut microbiota in subjects with obesity. Interestingly, all subjects before the intervention and after 2 months with placebo showed similar low microbial diversity, whereas the GTG showed a notable increase in diversity according to the Shannon index (figure 1A) and the unweighted and weighted beta diversity analysis (figure 1B,C). Furthermore, genistein also affected the abundance of the microbial communities (figure 1D). From the GreenGenes database, the taxonomic analysis of 16S rRNA gene sequencing revealed that before the intervention, Actinobacteria, Bacteroidetes, Firmicutes, Proteobacteria and Verrucomicrobia represented $98.2 \%$ of the total sequences at the phylum level. In these subjects, the Firmicutes:Bacteroidetes ratio was 1.07. After the intervention, the PG maintained a similar pattern, where the abundance of the five main phyla represented $97.9 \%$ of the total sequences, and the Firmicutes:Bacteroidetes
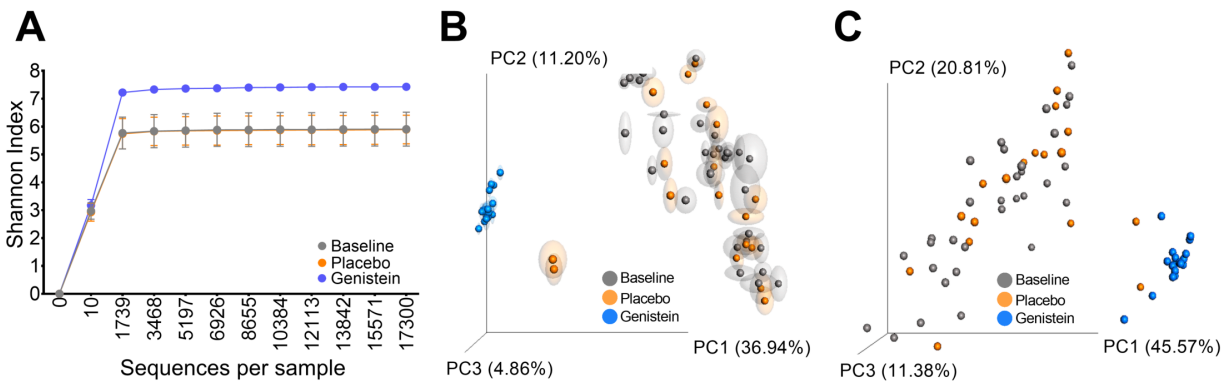

D
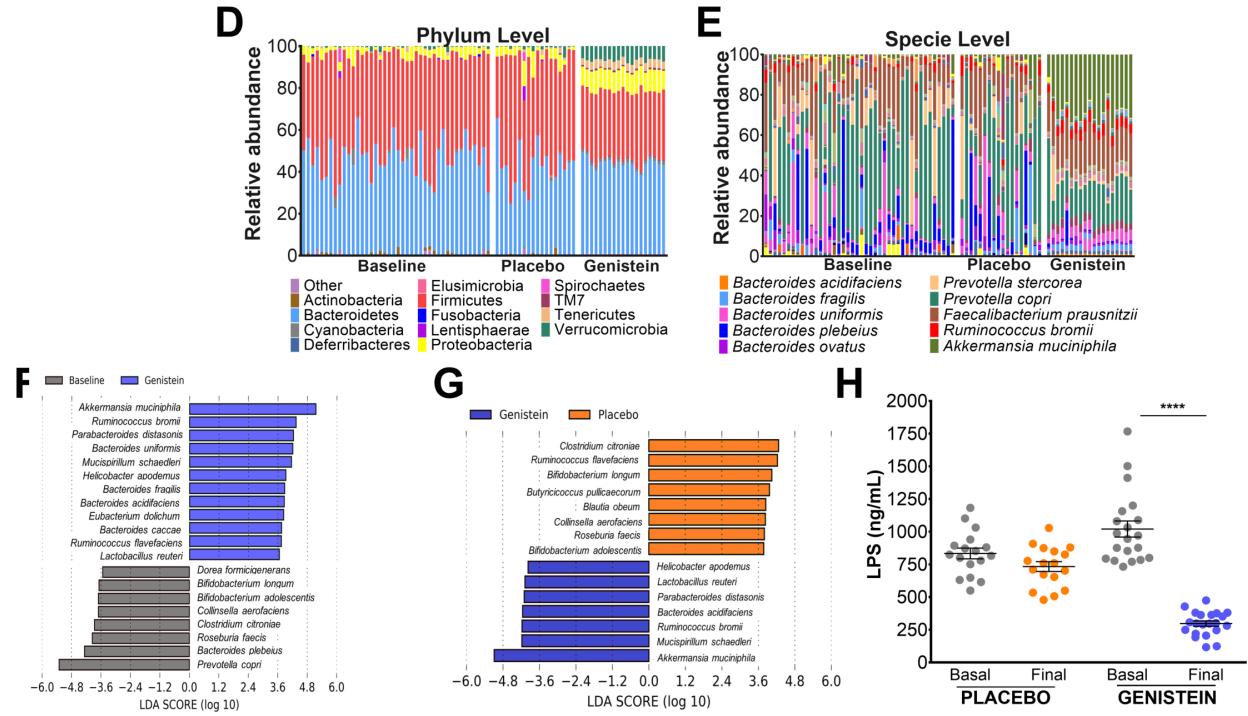

Figure 1 Analysis of fecal samples from genistein-treated humans reveals protection of metabolic endotoxemia. (A) Alpha diversity by Shannon index with rarefaction curve and beta diversity by principal coordinates (PC) analysis based on (B) unweighted or (C) weighted distances. The gray squares represent baseline samples; orange represents the placebo group; and blue represents the subjects that consumed genistein. (D) Taxonomic summary of the gut microbiota at the phylum and (E) species level. $(F, G)$ Taxa detected as differentially abundant by LEfSe analysis colored by groups and (H) serum LPS levels in subjects that consumed placebo or genistein. Values are given as mean \pm SEM $(n=22-23)$. Differences between the two groups were evaluated by paired t-test. ${ }^{* \star *} \mathrm{P}<0.0001$. LDA, linear discriminant analysis; LEfSe, linear discriminant analysis effect size; LPS, lipopolysaccharide. 

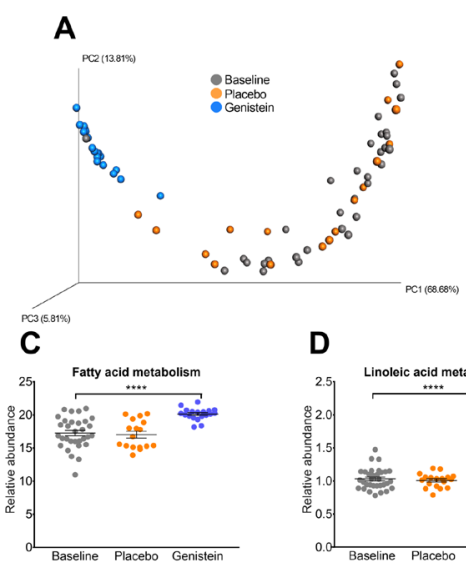

D
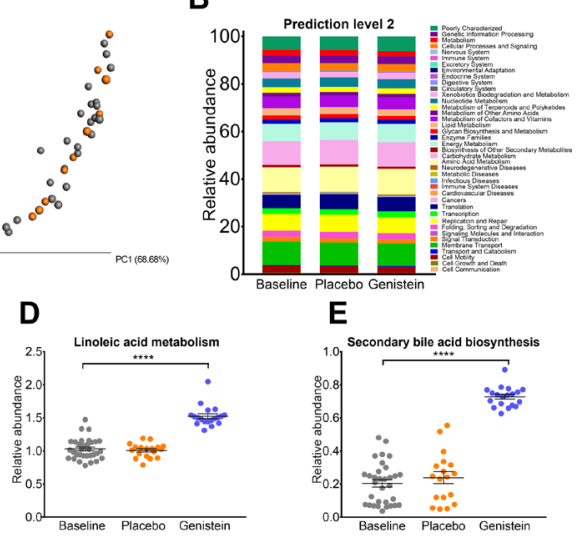

E
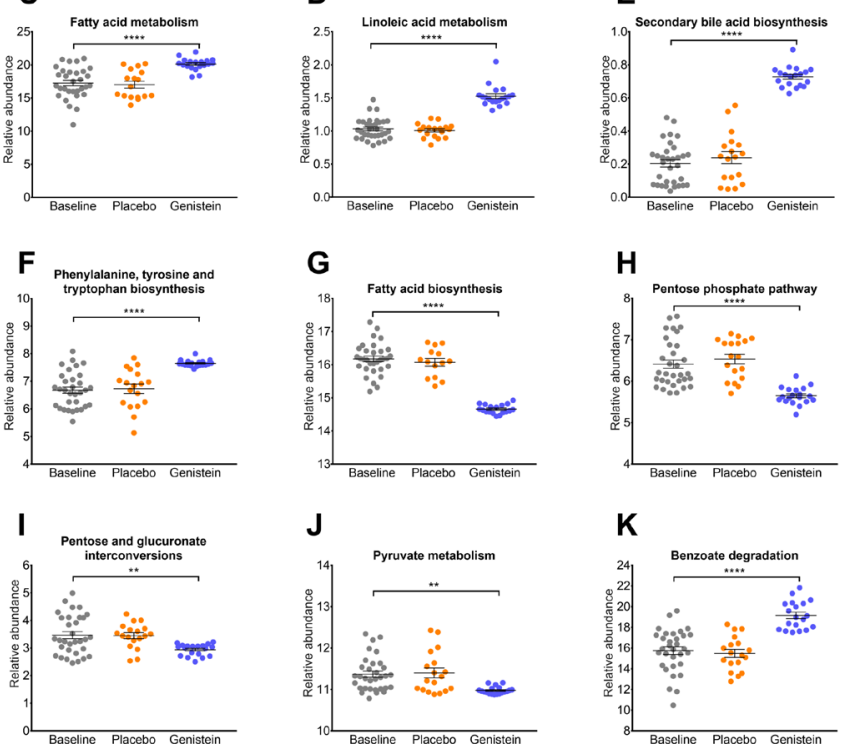

Figure 2 Metagenomic prediction by PICRUSt in humans that consumed placebo or genistein. (A) Principal component (PC) analysis plot comparing KEGG module predictions using $16 \mathrm{~S}$ data with PICRUSt. The gray circles represent baseline samples; orange represents the placebo group; and blue represents the subjects that consumed genistein.

(B) Relative abundances for specific KEGG modules. (C) Fatty acid metabolism; (D) linoleic acid metabolism; (E) secondary bile acid biosynthesis; $(F)$ phenylalanine, tyrosine and tryptophan biosynthesis; $(\mathrm{G})$ fatty acid biosynthesis; $(\mathrm{H})$ pentose phosphate pathway; (I) pentose and glucoronate conversions; (J) pyruvate metabolism; and (K) benzoate degradation. Values are given as mean \pm SEM $(n=22-23)$. Differences between the two groups were evaluated by paired t-test. ${ }^{\star \star} \mathrm{P}<0.01,{ }^{\star \star \star *} \mathrm{P}<0.0001$. KEGG, Kyoto Encyclopedia of Genes and Genomes; LEfSe, linear discriminant analysis effect size; PICRUSt, Phylogenetic Investigation of Communities by Reconstruction of Unobserved States.

ratio was 1.20. Interestingly, Actinobacteria, Bacteroidetes, Firmicutes, Proteobacteria and Verrucomicrobia represented $92.5 \%$ of the total sequences in the GTG. Genistein produced three important changes: an increase in low abundant phyla, including Cyanobacteria, Deferribacteres and Tenericutes, a decrease in the Firmicutes:Bacteroidetes ratio to approximately 0.76 due to a decrease in Firmicutes, and an increase in Verrucomicrobia from approximately $0.6 \%-0.9 \%$ to $6.78 \%$ (figure $1 \mathrm{D}$ ).

The taxonomic analysis at the genus level showed that the gut microbiota was represented by 60 genera. Fecalibacterium, Roseburia, Prevotella and Bacteroides represented $69.5 \%$ of the total genera in all subjects before the intervention. After the intervention, those four genera represented $65.4 \%$ of the total genera in the PG and only $35.5 \%$ in the GTG. Interestingly, five genera, including Paraprevotella, Suterella, Anaeroplasma, Akkermansia and Oscillospira, were increased in the GTG, representing $41 \%$ of all genera, in contrast to the $7 \%-10 \%$ represented in the PG and in all subjects before the intervention (figure 1E).

The linear discriminant analysis (LDA) effect size showed that Prevotella copri and Bacteroides plebeius were the most abundant species in all subjects before the intervention. Ruminococcus bromii and B. uniformis were increased, but the most abundant species was $A$. muciniphila in the GTG (figure 1F). In addition, the abundance of gut microbiota species was different between the PG and the GTG after intervention (figure 1F,G). As a consequence of the dysbiosis that all subjects had before the intervention, all subjects showed metabolic endotoxemia according to their LPS serum levels, which were not modified in the PG. Notably, the GTG showed a reduction of $70.8 \%$ when compared with the basal concentration ( $\mathrm{p}$ value $<0.0001$ ) (figure 1H).

\section{Genistein increased some metabolic functions of the gut microbiota}

Prediction of the potential metagenome functions of the intestinal gut microbiota using the Phylogenetic Investigation of Communities by Reconstruction of Unobserved States (PICRUSt) ${ }^{23}$ revealed that the PG maintained the metabolic functions of the gut microbiota according to the PCoA analysis (figure 2A), where the metabolism of amino acids, lipids and carbohydrates represented approximately $55.4 \%$ of all the metabolic functions of the gut microbiota in all groups (figure 2B). The metabolism of fatty acids significantly increased in the GTG, particularly linoleic acid metabolism ( $p$ value $<0.0001$ ) and the biosynthesis of secondary bile acids ( $p$ value $<0.0001$ ) and several amino acids, including phenylalanine, tyrosine and tryptophan ( $\mathrm{p}$ value $<0.0001$ ) (figure $2 \mathrm{C}-\mathrm{F}$ ). In contrast, there was a decrease in fatty acid biosynthesis ( $p$ value $<0.0001$ ) and the pentose phosphate pathway ( $\mathrm{p}$ value $<0.0001$ ) as well as in pyruvate metabolism ( $\mathrm{p}$ value $=0.002$ ) (figure $2 \mathrm{G}-\mathrm{J}$ ) were found. Notably, the metabolism of benzoate also increased with genistein ( $p$ value $<0.0001$ ) (figure $2 \mathrm{~K}$ ).

\section{Genistein modifies the abundance of some circulating phenolic metabolites derived from the gut microbiota}

Since genistein clearly induced a change in the metabolic activity of the gut microbiota, we assessed the presence of some metabolites derived from its metabolism (figure 3A). The analysis revealed that the GTG had higher levels of 4-ethylphenylsulfate, methyl-4- hydroxybenzoate and 3-phenylpropionate (figure 3B). Some of these metabolites have been associated with aerobic capacity, particularly with maximal $\mathrm{VO}_{2}$ after exercise, ${ }^{24}$ indicating an improved oxidative capacity of muscle. 

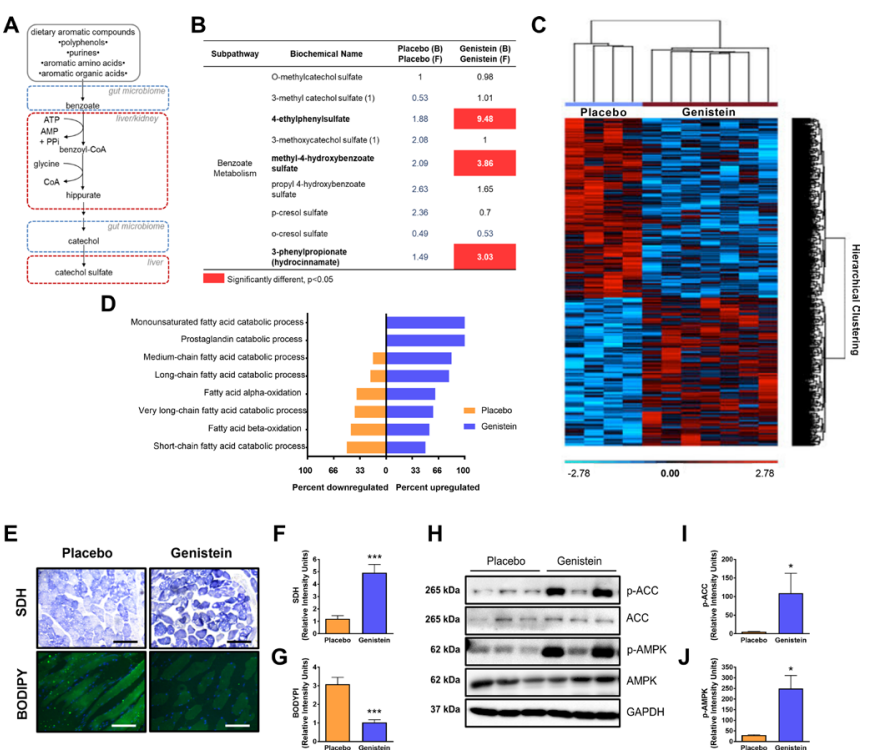

Figure 3 Genistein improves the oxidative capacity of skeletal muscle in subjects with obesity and insulin resistance (HOMA index greater than 2.5) after 2 months of treatment. $(A, B)$ Metabolomic analysis of the compounds derived from the benzoate metabolism of bacteria from the gut microbiota assessed by mass spectrometry at basal (B) and final $(\mathrm{F})$ assessment. (C) Hierarchical clustering heat map showing the relative intensity of the 376 differentially expressed genes (206 upregulated and 170 downregulated) in the GTG versus the PG. Analysis using Partek genomics suite; blue indicates downregulated expression and red indicates upregulated expression, with intensity of color reflecting intensity of upregulation or downregulation.

(D) Comparison of the percentage of upregulated and downregulated genes associated with fatty acid oxidation in the GTG versus the PG. (E) Microscopic analysis of SDH staining (up) and BODIPY 493/503 staining (down) showing mitochondrial activity and lipid accumulation, respectively, in sections of OCT-embedded skeletal muscle biopsies from the GTG and PG. The nuclei were stained with DAPI. The bar measures $200 \mu \mathrm{m}$ in size. (F) Densitometric quantitation of SDH staining and $(\mathrm{G})$ BODIPY staining using ImageJ. $(\mathrm{H})$ Immunoblotting analysis of total ACC, p-ACC, total AMPK and $p$-AMPK of skeletal muscle biopsies of obese patients in GTG and PG. (I) Densitometric quantitation of p-ACC/ total ACC and $(\mathrm{J}) \mathrm{p}-\mathrm{AMPK} /$ total AMPK. values are given as mean \pm SEM $(n=10)$. Differences between the two groups were evaluated by unpaired t-test. ${ }^{*} \mathrm{P}<0.05$, ${ }^{\star \star *} \mathrm{P}<0.001$. ACC, acetyl-CoA carboxylase; AMPK, 5'-adenosine monophosphate-activated protein kinase; BODIPY, borondipyrromethene; DAPI, 4',6-diamidino-2-phenylindole; GAPDH, glyceraldehyde 3-phosphate dehydrogenase; GTG, genistein-treated group; HOMA, Homeostatic Model Assessment; OCT, Optimal Cutting Temperature reagent; $\mathrm{p}$ ACC, Phospho-Acetyl-CoA Carboxylase; p-AMPK, PhosphoAMP-activated protein kinase; PG, placebo group; PPi, Pyrophosphate; SDH, succinate dehydrogenase.

\section{Genistein consumption increases the expression of genes involved in fatty acid oxidation in skeletal muscle}

In fact, a gene expression microarray analysis in skeletal muscle biopsies revealed that the expression of 206 genes was upregulated in the GTG (figure 3C). Genes associated with fatty acid oxidation, including those regulated by AMPK, were among the genes upregulated by genistein (figure 3D). In addition, the skeletal muscle of individuals in the GTG presented increased SDH staining, indicative of higher mitochondrial activity compared with that in the PG ( $p$ value $<0.0002$ ) (figure $3 \mathrm{E}, \mathrm{F}$ ). As a consequence, the skeletal muscle of individuals in the GTG showed a lower accumulation of triglycerides than that in those from the PG ( $p$ value $<0.0002$ ) (figure 3E,G), suggesting an increase in fatty acid oxidation. Furthermore, an enrichment pathway analysis derived from the microarray analysis revealed that genistein was associated with a main effect on AMPK, a central node that can stimulate several metabolic pathways, including fatty acid oxidation. In fact, genistein increased AMPK phosphorylation on the Thr 172 residue of the $\alpha$ subunit by 7.2-fold ( $\mathrm{p}$ value $=0.02$ ) (figure $3 \mathrm{H}, \mathrm{I}$ ) and ACC phosphorylation by 18.8-fold ( $\mathrm{p}$ value $=0.02$ ) (figure $3 \mathrm{H}, \mathrm{J}$ ) in the muscles of individuals in the GTG, suggesting an increase in fatty acid oxidation.

\section{Genistein increases the circulating concentration of fatty acid} oxidation metabolites

Furthermore, a metabolomic analysis of circulation showed increased free fatty acids, including mediumchain, long-chain and polyunsaturated fatty acids, in the GTG when compared with baseline or the PG (figure 4A), indicative of high-energy demand, as observed by an increase in dicarboxylic fatty acids that are $\omega$-fatty acid oxidation intermediates and hydroxy fatty acids that are formed during the intermediate stages of fatty acid oxidation $(p$ value $=0.01)$ (figure $4 \mathrm{~A}-\mathrm{D})$. Moreover, the increase in acylcarnitines and 3-hydoxybutyrate in the GTG was associated with increased $\beta$-fatty acid oxidation (figure 4A,E). Therefore, the observed changes are indicative of a signature of increased lipid metabolism with genistein supplementation that consists of augmented fatty acid mobilization and oxidation. These results suggest that the beneficial effects of genistein on the stimulation of fatty acid oxidation in skeletal muscle could be associated with an improvement in insulin sensitivity.

\section{Genistein consumption improves glucose tolerance}

Then, to assess the effect of genistein on glucose tolerance, we performed an oral glucose tolerance test (OGTT) on the subjects at baseline and at 2 months after treatment. The basal response was similar between groups, and after 2 months of intervention, the PG maintained the same response to the OGTT (figure 5A,B). Interestingly, the GTG showed a significant decrease, particularly in the serum insulin concentration, with respect to the basal OGTT ( $p$ value $=0.02$ ). The glucose and insulin AUC showed a $4.5 \%$ and $19.4 \%$ decrease, respectively, although a significant difference was reached only for insulin (figure 5C,D). As a consequence, there was an improvement of the HOMA index and the Matsuda index in the GTG (figure 5E,F), indicating an increase in insulin sensitivity. 
A

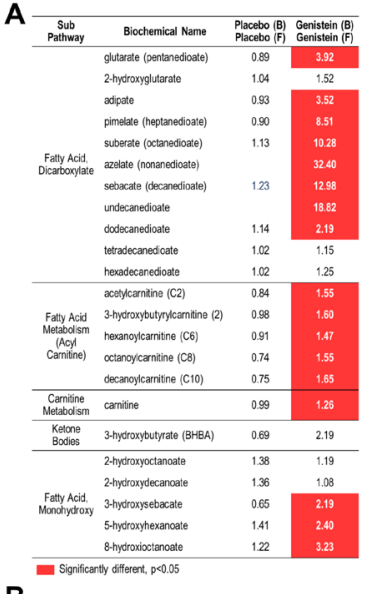

B

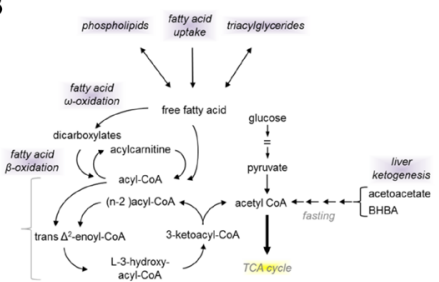

C

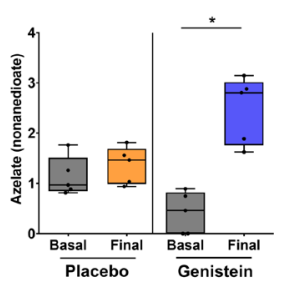

D

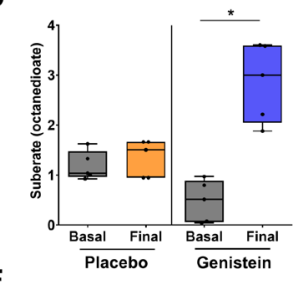

E

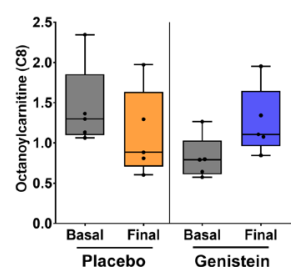

Figure 4 Genistein increases serum circulating acylcarnitines and dicarboxylic, hydroxy and free fatty acids, indicating the stimulation of fatty acid oxidation in skeletal muscle. (A) Metabolomic analysis in circulation of the individuals in the GTG when compared with baseline or the $P G(n=22-23)$. (B) Schematic of the biochemical pathways of lipids involved in the metabolomic analysis. (C) Azeleate, (D) suberate and $(E)$ octanoylcarnitine circulating concentrations during basal (B) and final (F) assessments of the GTG and the PG before and after the 2-month intervention $(n=22-23)$. Values are given as mean \pm SEM $(n=22-23)$. Differences between the two groups were evaluated by paired t-test. ${ }^{*} \mathrm{P}<0.05$. BHBA, beta-hydroxybutyrate; GTG, genisteintreated group; PG, placebo group.

\section{DISCUSSION}

Insulin resistance is a common alteration developed by subjects with obesity, and this is a consequence in part due to the development of lipotoxicity in non-adipose tissues, particularly the skeletal muscle. ${ }^{25}$ Several pharmacological treatments have been designed to attend this harmful problem in order to prevent the appearance of type 2 diabetes. Metformin, a plant-derived drug that was chemically modified, has been extensively used to reduce insulin resistance, and it has been demonstrated that its effects are mediated by the activation of AMPK ${ }^{26} 27$ as well as by modifying the gut microbiota. ${ }^{28}$ In the present study, similarly, we found that the soy isoflavone genistein has similar effects that also involve modifications of the gut microbiota and activation of AMPK.

Genistein was first identified in $1899,{ }^{29}$ and in 1931 , it was found that it is one of the three main isoflavones present in soybean. ${ }^{30}$ Several biological roles have been assigned to this molecule, especially that it was associated with a weak estrogen. ${ }^{31}$ However, in recent years, other biological activities have been associated with this compound, particularly with glucose metabolism. Several meta-analyses have

\section{Placebo}
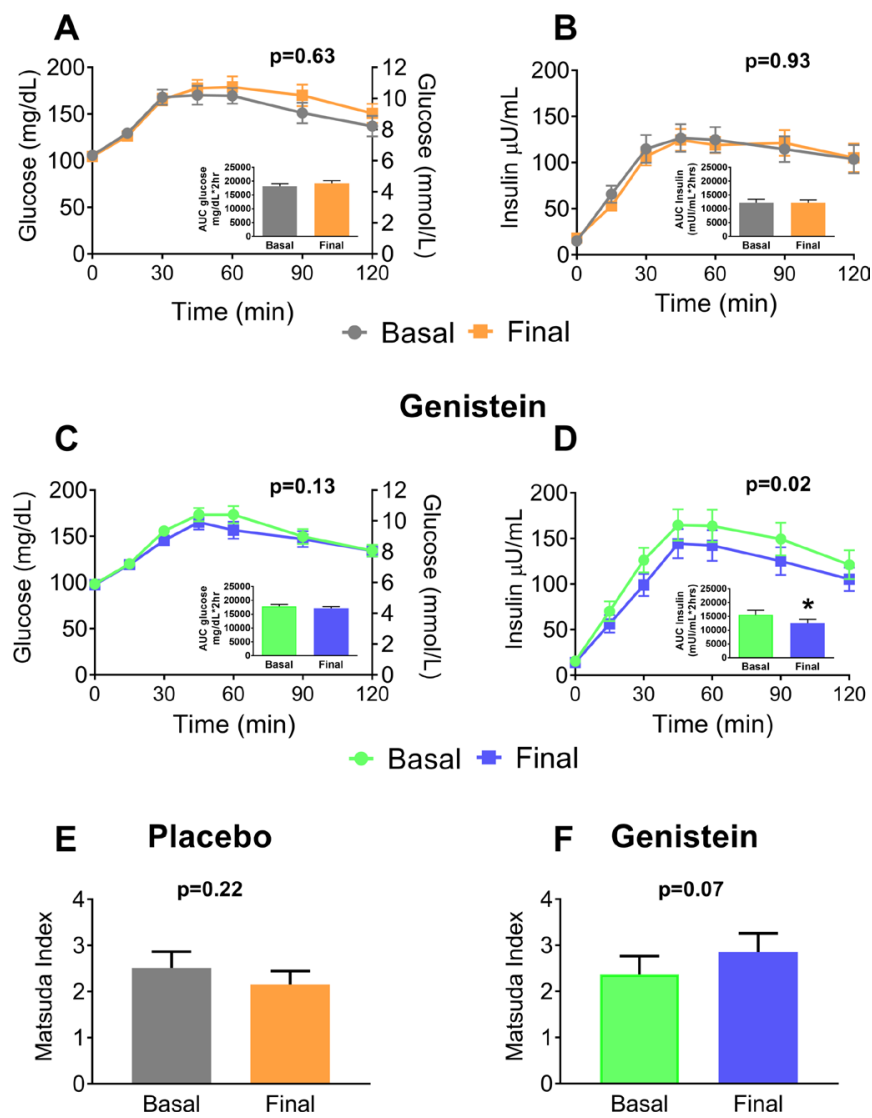

Figure 5 Genistein improves insulin sensitivity in subject with obesity and insulin resistance (HOMA index greater than 2.5). (A) Glucose and (B) insulin concentration during an oral glucose tolerance test in PG, comparing the levels before and after 2 months of placebo treatment. (C) Glucose and (D) insulin concentration during an oral glucose tolerance test in the GTG, comparing the levels before and after 2 months of genistein treatment. (E,F) Matsuda index in the PG and GTG before and after 2 months of treatment. Values are given as mean \pm SEM $(n=22-23)$. Differences between the two groups were evaluated by paired t-test. ${ }^{*} \mathrm{P}<0.05$. AUC, area under the curve; HOMA, Homeostatic Model Assessment; GTG, genistein-treated group; PG, placebo group.

confirmed that isoflavones improve fasting glucose and insulin levels, as well as insulin resistance. ${ }^{32} 33$ It has been suggested that soy isoflavones decrease the risk of type 2 diabetes in obese women. ${ }^{34}$ Studies in rats and C2C12 myotubes cells have demonstrated that genistein can activate the enzyme AMPK by increasing fatty acid oxidation in skeletal muscle, leading to an improvement in insulin sensitivity. ${ }^{12}$ Interestingly, genistein also stimulates AMPK in mouse hepatocytes and activates pathways involved in the cholesterol excretion. ${ }^{35}$ The present study demonstrates that in humans with metabolic syndrome and insulin resistance, consumption of genistein for 2 months improved significantly the insulin sensitivity in these subjects. It has been shown that the serum metabolite profile is a factor that has been associated with modifications in insulin sensitivity ${ }^{3637}$ In fact, our data showed that part of the beneficial effect of genistein took place by an increase in the oxidation 
of fatty acids as observed by the circulating metabolomics signature present in these subjects. These effects were associated by an increase in the oxidative capacity of the skeletal muscle on fatty acids, in part mediated by the activation of AMPK. Interestingly, in the present study, we demonstrated that the effects of genistein on carbohydrate metabolism might occur via a complex interaction involving the gut microbiota.

It is known, since the first description by Cani et al, that diabetes and obesity are characterized by the presence of metabolic endotoxemia, a condition that is dependent on circulating LPS levels. ${ }^{14}$ The elevation of LPS concentration has been associated to several mechanisms including the consumption of high fat in the diet or a change in the intestinal permeability. ${ }^{14} 38$ In fact, it has been demonstrated that dysbiosis of the gut microbiota can alter the gut permeability, and several factors can contribute to modifications of the gut microbiota, such as intestinal infections by pathogens, the use of antibiotics or dietary factors leading to the development of metabolic endotoxemia. ${ }^{15}$ The elevation of LPS is associated with a low-grade inflammation state, and this condition is frequently associated with the development of obesity. ${ }^{14}$ Interestingly, in the present study, we observed that genistein decreased the concentration of systemic inflammatory markers, in particular $\mathrm{C}$ reactive protein. In the last decade, it has been established that elevation of inflammatory markers can induce insulin resistance. ${ }^{40}$ Therefore, the elevation of fatty acid oxidation in skeletal muscle and the decrease in the inflammatory response due to the consumption of genistein are probably mainly responsible for the beneficial effects of this isoflavone on improving insulin resistance.

In our study, we found that genistein notably increased the phylum Verrucromicrobia in all subjects compared with those treated with placebo. The analysis revealed that the increase in the Verrucomicrobia phylum was associated with an increase in the Akkermansia genus. In particular, at the species level it was associated with an increase of A. muciniphila. Studies by Cani et al have revealed that an increase in A. muciniphila abundance is associated with lower blood glucose levels as well as low HOMA-IR in mice and humans. ${ }^{41-43}$

Interestingly, the PICRUSt analysis showed that genistein consumption can influence the bacterial metabolism of the gut microbiota, selectively increasing several metabolic pathways of the gut microbiota. It is of interest that some metabolites from the bacterial metabolism can influence some specific mechanisms associated with the use of fatty acids as energy sources. It has also been shown that genistein increases the formation of metabolites derived from the benzoate metabolism. In fact, there is evidence that genistein can be biotransformed by the gut microbiota to 4-ethylphenol. ${ }^{44}$ In particular, 4-ethylphenylsulfate, a product derived from 4-ethylphenol, is associated with the maximal aerobic capacity in healthy subjects, as well as prediction of lean body mass. ${ }^{24} 45$ Although 4-ethylphenylsulfate in a study was associated with the development of some neurobiological alterations, ${ }^{46}$ some studies have indicated that genistein can improve some neurodegenerative disorders. ${ }^{47}$ In fact, experimental evidence in mice also show that genistein can reduce the metabolic endotoxemia, improving the cognitive function. ${ }^{19}$ Therefore, the metabolic context of the subjects may influence the effects of several metabolites produced by the gut microbiota.

The results of the present study demonstrated that consumption of genistein in subjects with metabolic syndrome and insulin resistance can improve insulin sensitivity, an effect associated with a modification of the gut microbiota by increasing particularly A. muciniphila, and an increase in oxidative capacity of fatty acids in the skeletal muscle. Further studies are still needed to evaluate the long-term effect as well as the doses of genistein to improve glucose metabolism in humans, as well as if the effect is sustained on the gut microbiota after the removal of genistein. Our results show that the search of new molecules to deal with metabolic alterations associated with obesity is an important area of research, particularly for the treatment of insulin resistance to reduce the risk to develop type 2 diabetes.

Author affiliations

${ }^{1}$ Fisiologia de la Nutricion, Instituto Nacional de Ciencias Medicas y Nutricion Salvador Zubiran, Mexico

${ }^{2}$ Cirugía Experimental, Instituto Nacional de Ciencias Medicas y Nutricion Salvador Zubiran, Mexico

${ }^{3}$ Nefrología, Instituto Nacional de Ciencias Medicas y Nutricion Salvador Zubiran, Mexico

${ }^{4}$ Unidad de Microarreglos, Instituto Nacional de Medicina Genómica, Mexico

Contributors ART developed the concept. MG-C, NT, and ART designed the clinical study. ETG-S, EP-0, RG-H, LA-S, and MG-C conducted the clinical study. GT-V performed the surgeries to obtain biopsies. MS-T performed the fecal gut microbiota analysis. RM-E, MG-C, AL-B, and RG-H conducted the microarray analysis. RG-H, OG, MG-C, IT-V, and LGN performed biochemical and molecular analyses. MG-C, MS-T, GG, RM-E, AS-C, AV-C, NT, and ART conducted the statistical analysis and interpreted the data. MG-C, AV-C, and NT generated the figures and tables. ART wrote the manuscript. The final manuscript was approved by all authors.

Funding This work was supported by the Consejo Nacional de Ciencia de Tecnología (México) (grant number 261843 to ART).

Competing interests MG-C, NT and ART are inventors of the patent application (number MX/a/2018/003668) filed with the Mexican Patent Office on the use of genistein as a modulator of the gut microbiota, leading to beneficial effects in obese subjects.

\section{Patient consent for publication Not required.}

Ethics approval This protocol was approved by the ethics committee of the Instituto Nacional de Ciencias Medicas y Nutricion Salvador Zubiran (reference 1099).

Provenance and peer review Not commissioned; externally peer reviewed.

Data availability statement Data are available upon reasonable request. Data are available upon request to the correspondent author.

Open access This is an open access article distributed in accordance with the Creative Commons Attribution Non Commercial (CC BY-NC 4.0) license, which permits others to distribute, remix, adapt, build upon this work non-commercially, and license their derivative works on different terms, provided the original work is properly cited, appropriate credit is given, any changes made indicated, and the use is non-commercial. See: http://creativecommons.org/licenses/by-nc/4.0/.

\section{ORCID IDs}

Ivan Torre-Villalvazo http://orcid.org/0000-0001-7412-1153

Armando R Tovar http://orcid.org/0000-0002-1292-1312 


\section{REFERENCES}

1 Chooi YC, Ding C, Magkos F. The epidemiology of obesity. Metabolism 2019;92:6-10.

2 Head GA. Cardiovascular and metabolic consequences of obesity. Front Physiol 2015;6:32.

3 Guevara-Cruz M, Flores-López AG, Aguilar-López M, et al. Improvement of lipoprotein profile and metabolic endotoxemia by a lifestyle intervention that modifies the gut microbiota in subjects with metabolic syndrome. J Am Heart Assoc 2019;8:e012401.

4 Koves TR, Ussher JR, Noland RC, et al. Mitochondrial overload and incomplete fatty acid oxidation contribute to skeletal muscle insulin resistance. Cell Metab 2008;7:45-56.

5 Collier CA, Bruce CR, Smith AC, et al. Metformin counters the insulin-induced suppression of fatty acid oxidation and stimulation of triacylglycerol storage in rodent skeletal muscle. Am J Physiol Endocrinol Metab 2006;291:E182-9.

6 Zeng H-liang, Huang S-ling, Xie F-chun, Zeng HL, Xie FC, et al. Yhhu981, a novel compound, stimulates fatty acid oxidation via the activation of AMPK and ameliorates lipid metabolism disorder in ob/ ob mice. Acta Pharmacol Sin 2015;36:343-52.

7 Jeon S-M. Regulation and function of AMPK in physiology and diseases. Exp Mol Med 2016;48:e245.

8 Lelliott C, Vidal-Puig AJ, Lipotoxicity V-PAJ. Lipotoxicity, an imbalance between lipogenesis de novo and fatty acid oxidation. Int $J$ Obes Relat Metab Disord 2004;28 Suppl 4:S22-8.

9 Shaw CS, Clark J, Wagenmakers AJM. The effect of exercise and nutrition on intramuscular fat metabolism and insulin sensitivity. Annu Rev Nutr 2010;30:13-34.

10 Sacks FM, Lichtenstein A, Van Horn L, et al. Soy protein, isoflavones, and cardiovascular health: an American heart association science Advisory for professionals from the nutrition Committee. Circulation 2006;113:1034-44.

11 Palacios-González B, Vargas-Castillo A, Velázquez-Villegas LA, et al. Genistein increases the thermogenic program of subcutaneous WAT and increases energy expenditure in mice. J Nutr Biochem 2019;68:59-68.

12 Palacios-González B, Zarain-Herzberg A, Flores-Galicia I, et al. Genistein stimulates fatty acid oxidation in a leptin receptorindependent manner through the Jak2-mediated phosphorylation and activation of AMPK in skeletal muscle. Biochim Biophys Acta 2014;1841:132-40.

13 Bäckhed F, Manchester JK, Semenkovich CF, et al. Mechanisms underlying the resistance to diet-induced obesity in germ-free mice. Proc Natl Acad Sci U S A 2007;104:979-84.

14 Cani PD, Amar J, Iglesias MA, et al. Metabolic endotoxemia initiates obesity and insulin resistance. Diabetes 2007;56:1761-72.

15 Cani PD, Bibiloni R, Knauf C, et al. Changes in gut microbiota control metabolic endotoxemia-induced inflammation in high-fat dietinduced obesity and diabetes in mice. Diabetes 2008;57:1470-81.

16 Cani PD, Osto M, Geurts L, et al. Involvement of gut microbiota in the development of low-grade inflammation and type 2 diabetes associated with obesity. Gut Microbes 2012;3:279-88.

17 Santos GA, Moura RF, Vitorino DC, et al. Hypothalamic AMPK activation blocks lipopolysaccharide inhibition of glucose production in mice liver. Mol Cell Endocrinol 2013;381:88-96.

18 Cardona F, Andrés-Lacueva C, Tulipani S, et al. Benefits of polyphenols on gut microbiota and implications in human health. $J$ Nutr Biochem 2013;24:1415-22.

19 López P, Sánchez M, Perez-Cruz C, et al. Long-Term genistein consumption modifies gut microbiota, improving glucose metabolism, metabolic endotoxemia, and cognitive function in mice fed a high-fat diet. Mol Nutr Food Res 2018;62:e1800313:1800313.

20 Muniyappa R, Lee S, Chen $\mathrm{H}$, et al. Current approaches for assessing insulin sensitivity and resistance in vivo: advantages, limitations, and appropriate usage. Am J Physiol Endocrinol Metab 2008;294:E15-26.

21 Leal-Díaz AM, Noriega LG, Torre-Villalvazo I, et al. Aguamiel concentrate from Agave salmiana and its extracted saponins attenuated obesity and hepatic steatosis and increased Akkermansia muciniphila in C57BL6 mice. Sci Rep 2016;6:34242.

22 Stępień M, Stępień A, Wlazeł RN, et al. Obesity indices and inflammatory markers in obese non-diabetic normo- and hypertensive patients: a comparative pilot study. Lipids Health Dis 2014;13:29.

23 Langille MGI, Zaneveld J, Caporaso JG, et al. Predictive functional profiling of microbial communities using 16S rRNA marker gene sequences. Nat Biotechnol 2013;31:814-21.
24 Lustgarten MS, Price LL, Logvinenko T, et al. Identification of serum analytes and metabolites associated with aerobic capacity. Eur $J$ Appl Physiol 2013;113:1311-20.

25 Unger RH, diseases L. Lipotoxic diseases.. Annu Rev Med 2002;53:319-36.

26 Fullerton MD, Galic S, Marcinko K, et al. Single phosphorylation sites in ACC1 and ACC2 regulate lipid homeostasis and the insulinsensitizing effects of metformin. Nat Med 2013;19:1649-54.

27 Rena G, Hardie DG, Pearson ER. The mechanisms of action of metformin. Diabetologia 2017;60:1577-85.

28 Sun L, Xie C, Wang G, et al. Gut microbiota and intestinal FXR mediate the clinical benefits of metformin. Nat Med 2018;24:1919-29.

29 Perkin AG, Newbury FG. LXXIX. - The colouring matters contained in dyer's broom (Genista tinctoria) and heather (Calluna vulgaris). J. Chem. Soc., Trans. 1899;75:830-9.

30 Genistin WED. An isoflavone glucoside) and its Aglucone, genistein, from soybeans. J Am Chem Soc 1941;63:3273-6.

31 An J, Tzagarakis-Foster C, Scharschmidt TC, et al. Estrogen receptor beta-selective transcriptional activity and recruitment of coregulators by phytoestrogens. J Biol Chem 2001;276:17808-14.

32 Fang $\mathrm{K}$, Dong $\mathrm{H}$, Wang $\mathrm{D}$, et al. Soy isoflavones and glucose metabolism in menopausal women: a systematic review and meta-analysis of randomized controlled trials. Mol Nutr Food Res 2016;60:1602-14.

33 Zhang Y-B, Chen W-H, Guo J-J, et al. Soy isoflavone supplementation could reduce body weight and improve glucose metabolism in non-Asian postmenopausal women--a meta-analysis. Nutrition 2013;29:8-14.

34 Nanri A, Mizoue T, Takahashi Y, et al. Soy product and isoflavone intakes are associated with a lower risk of type 2 diabetes in overweight Japanese women. J Nutr 2010;140:580-6.

35 González-Granillo M, Steffensen KR, Granados O, et al. Soy protein isoflavones differentially regulate liver $\mathrm{X}$ receptor isoforms to modulate lipid metabolism and cholesterol transport in the liver and intestine in mice. Diabetologia 2012;55:2469-78.

36 Palmer ND, Stevens RD, Antinozzi PA, et al. Metabolomic profile associated with insulin resistance and conversion to diabetes in the insulin resistance atherosclerosis study. J Clin Endocrinol Metab 2015;100:E463-8.

37 Pedersen HK, Gudmundsdottir V, Nielsen HB, et al. Human gut microbes impact host serum metabolome and insulin sensitivity. Nature 2016;535:376-81.

38 Bein A, Zilbershtein A, Golosovsky M, et al. Lps induces hyper-permeability of intestinal epithelial cells. J Cell Physiol 2017;232:381-90.

39 Harris K, Kassis A, Major G, et al. Is the gut microbiota a new factor contributing to obesity and its metabolic disorders? J Obes 2012;2012:879151

40 Johnson AMF, Olefsky JM. The origins and drivers of insulin resistance. Cell 2013;152:673-84.

41 Dao MC, Everard A, Aron-Wisnewsky J, et al. Akkermansia muciniphila and improved metabolic health during a dietary intervention in obesity: relationship with gut microbiome richness and ecology. Gut 2016;65:426-36.

42 Schneeberger M, Everard A, Gómez-Valadés AG, et al. Akkermansia muciniphila inversely correlates with the onset of inflammation, altered adipose tissue metabolism and metabolic disorders during obesity in mice. Sci Rep 2015;5:16643.

43 Depommier C, Everard A, Druart C, et al. Supplementation with Akkermansia muciniphila in overweight and obese human volunteers: a proof-of-concept exploratory study. Nat Med 2019;25:1096-103.

44 Paul B, Royston KJ, Li Y, et al. Impact of genistein on the gut microbiome of humanized mice and its role in breast tumor inhibition. PLoS One 2017;12:e0189756.

45 Lustgarten MS, Price LL, Phillips EM, et al. Serum predictors of percent lean mass in young adults. J Strength Cond Res 2016;30:2194-201.

46 Hsiao EY, McBride SW, Hsien S, et al. Microbiota modulate behavioral and physiological abnormalities associated with neurodevelopmental disorders. Cell 2013;155:1451-63.

47 Malinowska M, Wilkinson FL, Langford-Smith KJ, et al. Genistein improves neuropathology and corrects behaviour in a mouse model of neurodegenerative metabolic disease. PLoS One 2010;5:e14192. 